\title{
Rúbrica para evaluar el equilibrio sobre el caballo en niños con autismo Rubric for evaluating balance on the horse in children with autism
}

\author{
* Juan Vives Vilarroig, *Paola Ruiz-Bernardo, **Andrés García Gómez \\ $*$ Universidad aumel (España), **U niversidad de Extremadura(España)
}

\begin{abstract}
Resumen. Lostrastornos del espectro autista(TEA) son un conjunto detrastornos del neurodesarrollo caracterizados por presentar déficits significativosen la comunicación social y un patrón de conductasy de intereses restringidos y repetitivos. Recientementeen las clasificaciones internacionales deenfermedades, se han incluido los problemas de integración sensorial, en concreto, las conductasdehiper ehiporreactividad sensorial, como unasintomatología propiade losT EA. Losproblemas de integración sensorial en el autismo afectan ala modulación sensorial pero también son el origen de otras dificultades de propioceptivas y vestibulares relacionadascon el control postural y el equilibrio. En este artículo se presentaunaherramientaqueal mismo tiempo sirve como programadeintervención y como rúbricadeobservación delasmejoras del equilibrio, utilizando al os caballos como herramienta para laintervención. Se utilizaalos caball os yaque aportan unarelación emocional justificada por la biofilia y además su movimiento y cadencia propician la mejora del equilibrio. Por lo tanto, el objetivo de este trabajo es presentar el diseño y validación de un instrumento (rúbrica) paraser utilizado con un grupo de al umnos conTEA y evaluar su equilibrio. La metodologíautilizadaparalavalidación comprende dos partes. Por un lado, la revisión lógica real izada por un grupo de expertos, quienes respondieron a un cuestionario sobre lacalidad de losítems propuestos, y paramedir el porcentaje de acuerdo entre los jueces se utilizó el Coeficiente de Concordanciade Kappade Fleissqueevidenció un acuerdo de $85,71 \%$. Por otro lado, y posteriormente, se realizó larevisión empíricacon unamuestradeniños conTEA. EIAlfadeC ronbach señalaque lafiabilidad general de laescalade 0,978 lo que sugierelasolidez internadel cuestionario. Por último, los resultados desu utilización evidencian la eficaciadel instrumento validado, en tanto, haservido para realizar la valoración y el seguimiento evolutivo del equilibrio en losniños participantesen lamuestra objeto de estudio.
\end{abstract}

Palabrasclave: Autismo; Control postural; Equilibrio; Evaluación educativa; Rúbrica; Terapiaasistida con caballos.

\begin{abstract}
Autism spectrum disorders (ASD) are a group of neurodevelopmental disorders character ised by significant deficits in social communication and apattern of restricted and repetitive behaviours and interests. Recently, sensory integration problems, specifically sensory hyper- and hyper-reactive behaviours, have been included in theinternational classifications of diseases as asymptomatology of ASD. Sensory integration problemsin autism affect sensory modulation but are al so the origin of other proprioceptive and vestibular difficulties related to postural control and balance. Thisarticle presentsa tool that serves both as an intervention program and as an observation rubric for balance improvements, by using hor sesas an intervention tool. Horses are used because they provide an emotional relationship justified by biophilia and, in addition, their movement and cadencepromotethe improvement of balance. Therefore, the aim of thiswork isto present the design and validation of an instrument (rubric) to be used with agroup of students withASD. The methodology used for validation comprisestwo parts. $0 \mathrm{n}$ the one hand, the logical review carried out by a group of experts, who answered aquestionnaire on the quality of the proposed items. A greement among the judges was measured with the FleissK appaC oncordance Coefficient, which showed an agreement of $85.71 \%$. On the other hand, and subsequently, the empirical review wascarried out with asample of children withASD. Cronbach'sAlphaindicated an overall reliability of the scal e of 0.978 , thus suggestingtheinternal robustness of the questionnaire. Finally, the results of its use showed the effectiveness of the validated instrument, as it was succesfully used to carry out the assessment and evolutionary follow-up of the bal ance in the children participating in the sampleunder study.
\end{abstract}

Keywords:Autism; Postural control; Balancing; Educational assessment; Rubric; Horse assisted therapy.

\section{Introducción}

Los trastornos del espectro del autismo (TEA) son un conjunto de trastornos del neurodesarrollo caracterizados por presentar déficits significativos en la comunicación social y un patrón de conductas y de intereses restringidos y repetitivos. Las per sonas conTEA no presentan un patrón homogéneo en cuanto a la intensidad y la variabilidad de sus síntomas, pudiendo clasificarse, en función del apoyo requerido para el desenvolvimiento adecuado en la vida ordinaria, en tres grados de severidad (APA, 2013).

Fecha recepción: 07-12-20. Fecha de aceptación: 25-02-21

María Paola Ruiz Bernardo

ruizma@uji.es
LOSTEA se asocian frecuentemente a otros trastornos del neurodesarrollo y a otras condiciones médicas y se entiende que tienen un origen genético multifactorial aún sin clarificar de forma definitiva (Kalsner et al., 2018). En ocasiones vienen asociados con discapacidad intelectual (Baio et al., 2018) y el desarrollo de su lenguaje oral oscila desde la corrección formal hasta el mutismo (APA, 2013).

En los últimos diez años han pasado de ser considerados como una enfermedad rara, por su baja preval encia, a considerarse como un trastorno bastante común. Recientes estudios epidemiológicos señal an que cerca 1 de cada 59 niños menores de 8 años presentan sintomatología compatible con losTEA (M zenner et al., 2020). 
El Diagnóstico clínico de losTEA se establece a través de un equipo multidisciplinar altamente especializado en el que se deben recoger aspectos médicos y conductuales con laaplicación de pruebas estandarizadas, generalmente conADI-R (Rutter et al., 2006) yADOS2 (Lord et al., 2008), que recogen de forma sistemática la sintomatología referida a los criterios de diagnóstico establecidos en los manuales de clasificación de enfermedades tales como DSM-5 (APA, 2013) o CIE, 11 (W HO, 2019). Además del juicio clínico la evaluación debe contemplar otros aspectos complementarios referidos tanto a la sintomatología esencial del síndrome como a otros aspectos comórbidos o relacionados con la situación de discapacidad que genera el trastorno y con la evaluación del contexto socio-familiar que rodea a la persona conTEA.

El tratamiento del autismo basado en la evidencia es eminentemente educativo, de intervención conductual intensiva y temprana, a veces acompañado de prescripciones farmacológicas para el tratamiento puntual de síntomas asociados tal es como la ansiedad, las conductas desafiantes o los trastornos obsesivos compulsivos, entre otros (Sam et al., 2019; W illiamson et al., 2017).

U nos de los trastornos que aún no forman parte de la sintomatología esencial de síndrome, aunque suele acompañarlo, son los trastornos motores (Fournier et al. , 2010). En DSM -5 aparecen como unasintomatología asociada que ayuda a apoyar el diagnóstico (APA, 2013) y pueden considerarse como un indicador de pronóstico para la conducta adaptativa futura de la per sona conTEA (Stevenson et al., 2017). A pesar de no formar parte del cuadro esencial del síndrome, algunos autores consideran que deben siempre contemplarse en la evaluación del trastorno (Crespo-Eguílaz y Narbona, 2009; Naksakul; 2008).

Entre los trastornos motores más frecuentes en el TEA se encuentran los problemas de coordinación diná mica general, los problemas en la flexibilidad y en el tono motor, problemas en la coordinación motora fina, problemas en el control postural y los problemas en el equilibrio (Pollock, Durward, Rowe y Paul, 2000). En este ámbito de los problemas motores, los trastornos del equilibrio son muy frecuentes y además requieren una especial atención puesto que tienen una gran implicación en los desempeños de las actividades de la vida diaria (N aksakul, 2008). De hecho, las tareas cotidianas como salir de la ducha, cocinar en la estufa o vestirse se vuelven sustancialmente más complejas si no se puede confiar constantemente en la estabilidad durante la posición de pie (Fisher et al., 2018). Por otro lado, las dificultades de estabilidad postural se han relacionado con lagravedad de lossíntomas del autismo (Radonovich et al. 2013; Travers et al. 2013).

Se puede entender que el equilibrio es la capacidad que tiene nuestro cuerpo para mantenerse erguido a través de los movimientos compensatorios que realiza cuando está quieto o en movimiento (M osston, 1993). El equilibrio proporciona al organismo una base estable para la locomoción, las acciones manual es y las acciones faciales, facilitando una interacción eficaz con el entorno, tanto físico como social (Adolph \& Franchak, 2017).

El equilibrio es una conducta compleja en el que están implicados varios sistemas sensoriales. El sistema visual que aportainformación sobre la posición del cuerpo en relación con el espacio y los objetos (MontesCastillo, Pérez, Díaz-Barriga y Vázquez; 2000), el sistema vestibular que determina la posición de la cabeza con la gravedad y los movimientos de aceleración y desaceleración de ésta (Ayres, 2008), y el sistema propioceptivo, que interviene en los procesos de discriminación y localización de las partes del cuerpo, ayudando a modular la fuerza de contracción, la temporalización del movimiento y las reacciones de enderezamiento, entre otras ( Ricciardi, 2010).

La eficacia de los programas de entrenamiento de equilibrio para niños con autismo aún no se conoce bien y por lo tanto los resultadosno son aún concluyentes. Se conoce que en otras poblaciones diferentes al autismo (personas sanas, personas con lesiones cerebrales o personas de edad avanzada), los programas basados en la mejora del equilibrio mediante la estimulación de los sistemas sensoriales (visual, vestibular y propioceptivo implicados) han resultado exitosos(Francino, et al., 2019), pero en el autismo la investigación no es aún concluyente (Cheldavi et al., 2014).

En este contexto de provisionalidad científica, se han realizado investigaciones prometedoras para mejorar el equilibrio de las personas con autismo, entre otros, con programas de integración sensorial (Sam, Smith y Kai, 2017), através de videojuegos (Traverset al. , 2018), con programas de entrenamiento en artes marciales (Sarabzadeh, Azari \& Helalizadeh, 2019), con entrena miento gimnástico y entrenamiento físico (Akyol \& Pektas, 2018; Pan et al., 2017); y también con la ayuda de caballos (Portela-Pino, Bouzo-Gónzalez \& Pino-Juste, 2019).

Montar a caballo puede facilitar el control postural de las personas con autismo y con ello la mejora del equilibrio, puesto que en esta actividad se ponen en juego múltiples estímulos de diversos canales sensoriales 
(Hameury et al., 2010). Es más, los movimientos rítmicos que el caballo transmite al jinete y los cambios gravitacionales continuos que exigen reajustes posicionales inmediatos, hacen que montar a caballo sea una actividad con influencia muy positiva sobre el equilibrio, la coordinación y la postura (Bronson et al. 2010 ; M uñoz-Lasa et al. 2011; T seng et al. 2013), además de ser una actividad altamente motivadora (Kern et al, 2011; García-Gómez et al., 2014).

Los trabajos que han tratado de mejorar el equilibrio de las personas con autismo con la utilización de caballos se basan en la aplicación de programas desarrollados por monitores formados en distintos métodos generalistas, pero adolecen de la especificidad suficiente como para poder ser replicados con exactitud, lo cual abunda en la crisis de reproductibilidad achacada a la investigación científica actual (Nosek et al., 2015). La especificación y la estandarización de los programas de intervención es una realidad demandada en varias revisionessistemáticas(Trzmiel et al. , 2019; Charry-Sánchez et al. 2018).

Por otro lado, la evaluación de la eficacia de las intervenciones, tanto terapéuticas como educativas, exige de instrumentos de evaluación que permitan tanto evaluar el impacto de los programas como realizar un seguimiento continuo de los aprendizajes. Los estudios sobre las intervenciones con caballos han ido ganando en rigor en los últimos años, pero solo informan de instrumentos de evaluación del impacto de las terapias a través de observaciones conductuales estandarizadas (Grabriels et al. 2015) y también, de forma incipiente, a través de la utilización de biomarcadores (Tabares et al. , 2013), pero los estudios no informan sobre los instrumentos de evaluación que permitan observar el progreso de los participantes a lo largo de todo el progra ma educativo o reeducativo (Villalobos-Samaniego, 2019).

Las rúbricas como procedimiento de evaluación de los aprendizajes son instrumentos de evaluación de gran valor y cada vez más reconocidos por la comunidad educativa, demostrando su potencial en la orientación de los programas educativos y de los aprendizajes y mostrando a través de los estudios de confiabilidad, con un alto nivel de acuerdo entre los distintos evaluadores (Reddy y Andrade, 2010). Los estudios de confiabilidad entre evaluadores en los distintos estudios realizados oscilan entre moderados y altos, tanto para las evalua ciones grupales como para la individuales (Hafner \& Hafner, 2003). Además, las rúbricas aportan transpa rencia en el proceso de evaluación puesto que informan a los usuarios acerca de cuáles son los criterios de evaluación y de logro antes del proceso de aprendizaje (Jonsson, 2014).

Las rúbricas o matrices de valoración son instrumentos de evaluación que pueden adoptar diversas formas, pero básicamente todas ellas tienen una serie de elementos comunes: son tablas que desglosan los niveles de desempeño de los estudiantes en un aspecto determinado, con criterios específicos sobre rendimiento (Gatica-Lara \& U ribarren-Berrueta, 2013).

En el proceso de elaboración de una rúbrica se pueden distinguir una secuencia ordenada de acciones: 1) determinar objetivos del aprendizaje, 2) Identificar los elementos 0 aspectos a valorar, 3) D efinir descriptores, escal as de calificación y criterios, 4) Determinar el peso de cada criterio, y 5) Revisar la rúbrica diseñada, reflexionar sobre su impacto educativo y aportar en su caso indicadores de fiabilidad y validez (Reddy y Andrade, 2010; Gatica-Lara\& U ribarren-Berrueta, 2013; Martínez-Rojas, 2008).

Por lo tanto, se entiende que una rúbrica puede ser un procedimiento adecuado para evaluar el progreso de los participantes con autismo que siguen un programa con ayuda de caballos para la mejora del equilibrio.

Dicho lo anterior, el objetivo en el presente trabajo es presentar el proceso de elaboración y la valoración de una rúbrica destinada a evaluar las destrezas de equilibrio sobre el caballo perteneciente a un programa de intervención para niños con autismo.

\section{Material y métodos}

Para realizar el proceso de diseño y validación del instrumento se realizaron dos acciones de valoración secuenciadas en dos fases. Una revisión lógica, para valorar los ítems del instrumento piloto, y una revisión empírica, para analizar la aplicación del instrumento y así depurar y consolidar el cuestionario final. Además, para ver la efectividad del instrumento se utilizaron los mismos datos de validación. Es importante destacar que este mismo instrumento es a la vez una rúbrica de observación de la mejora del equilibrio y un programa progresivo de entrenamiento para dicha mejora utilizando un caballo. Esto condicionalas mediciones, en tanto, se observa una puntuación muy baja en el inicio del programa (test de diagnóstico) y un puntaje alto al final del programa (efectividad del programa).

A continuación, se detallan las dos fases de elaboración del instrumento. 


\section{Fase I: Revisión lógica}

Diseño: el instrumento fue objeto de una validación de instrumento mediante juicio de expertos (EscobarPérez \& Cuervo-Martínez, 2008; González, Sánchez \& Jornet, 2011), donde éstos valoraron la comprensión y la relevancia de los ítems del instrumento en relación a la medición y evolución del equilibrio.

Instrumento: el formulario de valoración que se utilizó para la consulta a los jueces constaba de una primera parte que incluía los datos per sonales y profesionales de los jueces y siete aspectos a evaluar del cuestionario. Estos siete aspectos eval uados hacen referencia a la redacción y a la pertinencia de los elementos a valorar de la rúbrica y a la escala de valoración.

Participantes: para que el equipo de expertos valorara adecuadamente el cuestionario inicial, al seleccionarlos tuvimos en cuenta qué queríamos medir y dónde lo íbamos a aplicar tal como sugieren Escobar-Pérez y Cuervo-Martínez (2008). Por ello el grupo de expertos estabaformado por seis personas: una fisioterapeuta, un instructor de equitación, una terapeuta ocupacional, una doctora en fisioterapia, una doctora en educación y un doctor en psicopedagogía, todos ellos en activo y con amplia experiencia en el tema de la intervención terapéutica asistida con caballos para personas con diversidad funcional.

Análisis de datos: para evaluar el grado de acuerdo entre los jueces se utilizó el estadístico kappa libremarginal para múltiples evaluadores de Randolph (Randolph, 2005, Warrens, 2010), que es una adaptación del Kappa de Fleiss (1971). Los valores de kappa pueden variar entre $-1,0$ y 1,0, siendo - 1,0 el perfecto desacuerdo por debajo del azar, 0,0 el acuerdo igual al azar y 1,0 el acuerdo perfecto por encima del azar. La regla empírica de Fleiss (1971) es que los valores de kappainferioresa 0,40 son «pobres», los valores de 0,40 a 0,75 son «intermedios a buenos», y los valores superiores a 0,75 son «excelentes». Para su cál culo se utilizó la calculadora en línea de Randolph (2008).

\section{Fase II: Revisión Empírica}

Diseño: Se ha seguido un diseño de tipo descriptivo cuyo objeto es corroborar el funcionamiento de losítems

\footnotetext{
Tabla 1.

Variabilidad utilizada en el instrumento. Fuente: elaboración propia.

0 Se desequilibra, los dos monitores de apoyo le sujetan con las dos manos, no puede

real izar el ejercicio o se tiene que parar el caballo porque se cae.
1 Se desequilibra continuamente, en tres o más ocasiones mientras dura el ejercicio, los dos monitores de apoyo le sujetan con las dos manos.

2 Se desequil ibra en al menos dos ocasiones, mientras dura el ejercicio, los dos monitores de apoyo le sujetan con las dos manos.

3 No se desequilibra, pero es necesario que los dos monitores de apoyo vayan cogiéndole 0 ayudándole con las manos en ambos laterales.

No se desequilibra, es suficiente que sea solo un monitor el que le apoye poniendo 0 ayudando con las manos.

5 No se desequilibra, los monitores no le sujetan.
}

y de la rúbrica en su conjunto.

Instrumento: el cuestionario piloto estaba formado por 84 ítems que se distribuía en 3 dimensiones (con el caballo en parado, al paso y al trote), se utilizó una esca la de 6 puntos que presentaban la siguiente variabilidad (ver tabla 1).

Participantes: Dado que este programa y rúbrica de observación ha sido creada para mejorar el equilibrio de niños conTEA. Lamuestrase haformado por 8 niños y 1 niña, entre 9 y 12 años conTEA. Los cuales una vez al mes durante 6 meses que duró el tratamiento realizaron los ejercicios que sugiere la rúbrica y finalizado el programa se obtuvieron 3612 anotaciones que conformaron la base de datos para los análisis realizados.

Análisis de datos: Se realizaron estudios de frecuencia, descriptivos y el Alfa de Cronbach. Y para establecer si ha sido efectiva la utilización de la rúbrica, tanto como programa de entrenamiento y como instrumento de medición, se ha utilizado un estadístico de diferencias de medias para dos muestras relacionadas (prueba no paramétrica) denominadaW ilcoxon.

\section{Resultados}

\section{Resultados de la Fase I: revisión lógica}

Como se ha dicho, para evaluar el grado de acuerdo entre los jueces se ha utilizado el Coeficiente de Concordancia de Kappa de Randolf (2005). El resultado de Kappa para los 7 ítems determina que existe un buen acuerdo entrejueces $(0,71)$ con un $85,71 \%$ de acuerdo.

\section{Resultados de la Fase II: revisión empírica}

Una vez revisada la rúbrica de observación tipo piloto se realizó una prueba empírica para observar el comportamiento de los ítems y de la rúbrica en general. Esto se realizó mediante la primera medición de los 9 niños que participaron del proyecto.

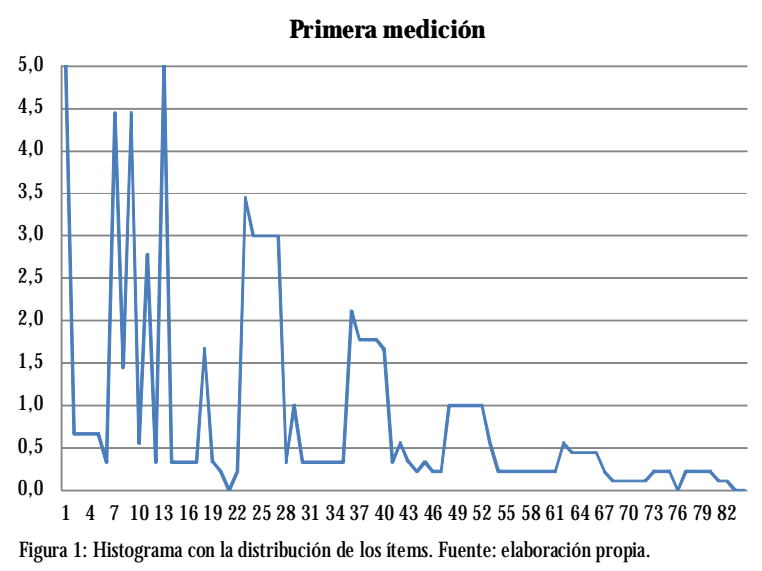


En cuanto a los datos descriptivos obtenidos de la primera medición, que se puede observar en el Anexo 1 , los registros tienen una tendencia por la cual desde un principio los primeros ítems puntúan más alto y los últimosítems menos altos. Esto es propio de la rúbrica, en tanto, vaescalonando el esfuerzo requerido para evolucionar en el dominio del equilibrio. Como podemos ver en la figura 1, donde se presenta un histograma con la distribución de los ítems.

En cuanto a los ítems mejor valorados en esta primeraval oración destacamosel 1 y el 13 , ya que su media general hace evidente que es una situación ya lograda desde el punto de partida. La media general es de 5, es decir que el $100 \%$ de los alumnos lo hacen la primera vez que montan en el caballo (ver tabla 2). Estos dicen:

Tabla 2.

Los ítems más valorados. Fuente: elaboración propia

$1 \quad$ Guarda el equilibrio con ojos abiertos, cogido al cinchuelo.

13 Guarda el equilibrio ojos abiertos sin cogerse del cinchuelo.

En estos casos se puede sugerir que al no presentar variabilidad ni al principio ni al final estosítems se pueden quitar de la rúbrica para evitar tener tantos distractores.

Los ítems menos valorados son claramente aquellos que exigen una mayor destreza en equilibrio, ellos son el 21, el 76, el 83 y el 84; en todos los casos ninguno de los alumnos ha podido hacerlo, en la evaluación inicial, por lo cual se ha valorado con un 0 (Ver tabla 3). Estos dicen:

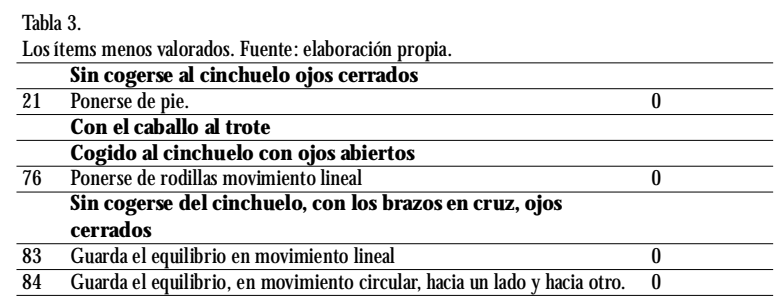

Al contrario de lo que sucede con los ítems mejor valorados (1 y 13) losmenos valorados (21, 76, 83 y 84) durante el progreso del programa mostrarán variabilidad por lo cual es recomendable que se mantengan dentro de la rúbrica.

Posteriormente, para observar la fiabilidad de la prueba se realizó un análisis general de la escala a través del Alfa de Cronbach y el resultado fue de 0,978, lo que nos sugiere la solidez interna del cuestionario. También se utilizó la misma prueba con cada uno de los ítems. Se observó que el Alfa de la escala en general no mejoraba significativamente si era eliminado algún ítem, ya que en todos los casos el Alfa fue superior a 0,97. Así pues, se puede apreciar que todos los ítems son importantes para valorar el equilibrio y que la rúbrica creada permite medir dicha destreza.

\section{Resultados de la eficacia del instrumento: uso de la rúbrica de observación para medir la evolu- ción del equilibrio}

El primer día que montaron los niños se utilizó la rúbrica para realizar un diagnóstico de cada uno de los niños. Posteriormente, una vez al mes, mientras duró el tratamiento, se recogieron los datos de observación con la rúbrica. Para poder anal izar los datos se comenzó real izando un sistema de agrupamiento, de manera que se obtuvieron las medias de los ejercicios que contenían los bloques «cogido al cinchuelo ojos abiertos, cogido al cinchuelo ojos cerrados, sin cogerse al cinchuelo ojos abiertos y sin cogerse al cinchuelo ojos cerrados».

De este modo se redujo los datos a cuatro grupos para el bloque de «el caballo en parado», cuatro para el bloque de «el caballo al paso» y cuatro para el bloque de «el caballo al trote».

A continuación, se volvió a reagrupar los datos haciendo medias y se obtuvo únicamente con un grupo de datos para ejercicios en parado, otro para ejercicios al paso y otro para ejercicios al trote.

Finalmente se hizo la media de estos tres grandes bloques y se obtuvo un único grupo de datos al que se le \|lamó «equilibrio sobre el caballo».

Posteriormente los datos se pasaron a gráficos y se realizó un análisis visual, para cada uno de los participantes de la muestra, acompañado de un análisis estadístico.

A continuación, se presentan los resultados de efica cia del tratamiento de los niños de la muestra, (ver figura 2).

En la figura 2 se observa cómo, en todos los participantes, se produce un cambio de nivel abrupto entre el dato de base y el primer dato de la línea de tratamiento, observándose también una línea de tendencia ascendente en todos los casos.

Visualmente se observa cómo ha habido una progresión y cómo, a lo largo del proceso de tratamiento, los alumnos han ido adquiriendo destrezas para poder realizar los ejercicios propuestos cada vez con menos ayudas por parte de los monitores de apoyo que acompañaron a los al umnos durante todo el proceso de intervención.

\section{Análisis estadístico grupal de las destrezas ad-} quiridas sobre el caballo

En la tabla 4 se presentan los estadísticos descripti- 


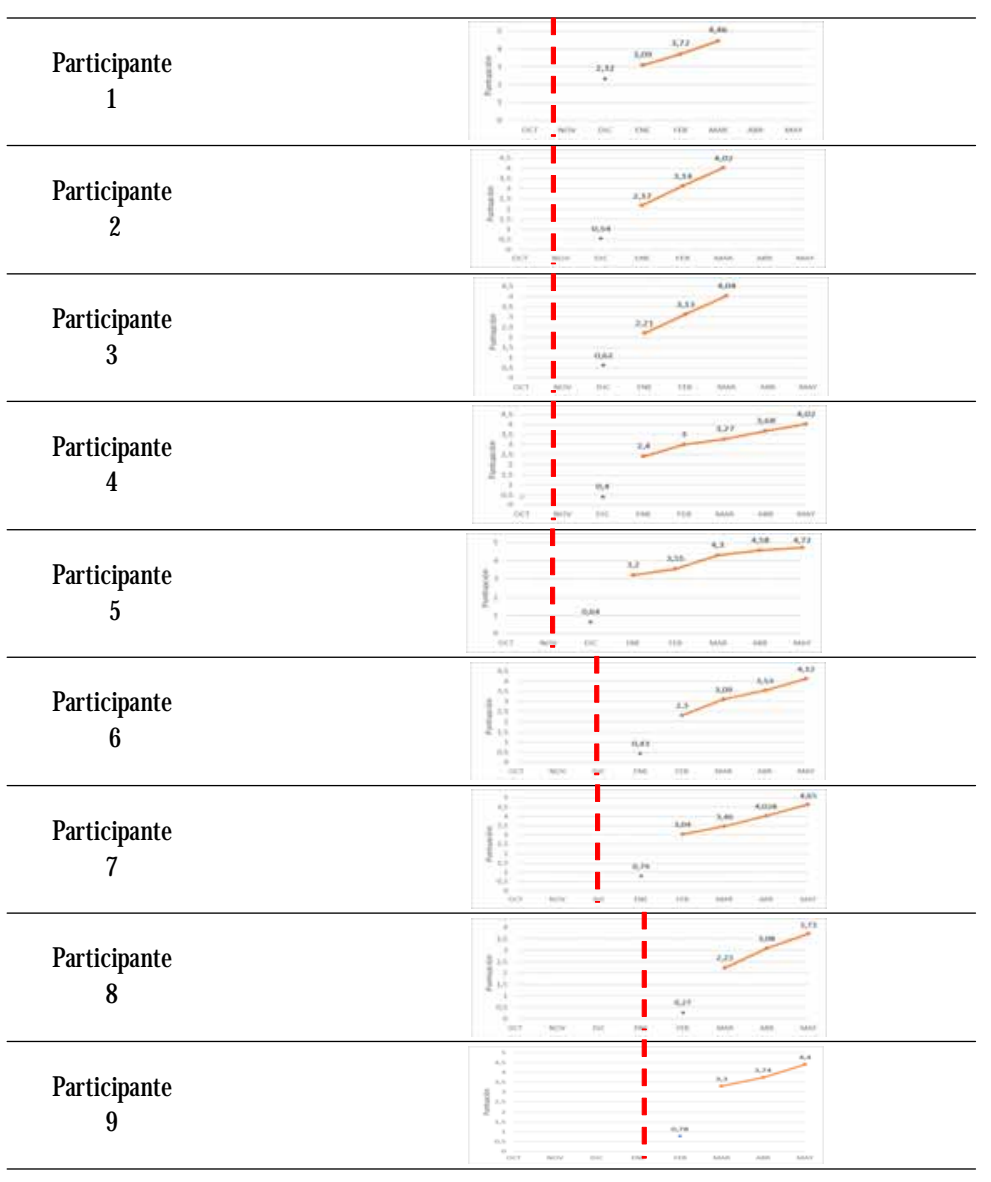

Figura 2. A nálisis visual grupal. Destrezas sobre el caballo. Fuente: Elaboración propia
Mosston (1993) y Adolph \& Franchak (2017), lo que demuestra la adecuada construcción del instrumento para abarcar la totalidad de posibilidades que integran el concepto de equilibrio.

Por otra parte, la revisión realizada mediante el juicio de expertos asegura un cuestionario de gran calidad y comprensión, algo que se ve apoyado al comprobar los datos de los altos porcentajes de Concordancia de Kappa de Randolf (85,71\%).

Del mismo modo, los excelentes resultados obtenidos en la prueba de fiabilidad, destacando un 0,978 en cuanto al Alfa de Cronbach, no hacen más que reforzar la valoración del cuestionario, poniendo de manifiesto su fiabilidad, algo que también podemos ver reflejado en los datos del ensayo empírico realizado.

Además, se ha podido valorar, a nivel visual y estadístico, el efecto que ha producido el programa educativo en el equilibrio de los participantes.

Estos resultados nos muestran que el efecto obtenido también ha oscilado en-

vosy de contraste referidosa las puntuaciones test retest de la muestra de participantes evaluados. Se puede observar cómo el efecto del tratamiento sobre el grupo de los nueve alumnos, expresado a través de la d de Cohen, es significativo y de magnitud de efecto grande $(p=.008 ; d=3.89)$. Del análisisdelaevolución del aprendizaje en las destrezas de equilibrio, se observa que los participantes han necesitado cada vez menos ayuda para realizar los ejercicios propuestos durante el programa de intervención.

Tabla 4.

Estadísticos descriptivos y de contraste de las puntuaciones test retest de los nueve participantes Fuente: elaboración propia.

\begin{tabular}{ccccc}
\hline Test M edia $\pm D . T$ & Retest Media \pm D.T. & $z$ & $p$ & $d$ \\
\hline $90.75 \pm 0.61$ & $94.24 \pm 0.33$ & 2.668 & 0,008 & 3.89
\end{tabular}

\section{Discusión y conclusiones}

Tras revisar la bibliografía referente al equilibrio y su relación con los niños afectados conTEA, se considera que el cuestionario creado tiene la suficiente solidez teórica para ser utilizado como instrumento de medida para el equilibrio sobre el caballo.

Cabe resaltar que su constitución definitiva recoge la totalidad de los rasgos del equilibrio definidos por tre medio y alto para la mayoría de los participantes, donde los sistemas: visual, vestibular y propioceptivo han estado funcionando de maneradestacada. Por lo tanto, se puede inferir que estos sistemas han experimentado una importante mejoría durante la fase de trata miento en los participantes. Tal como lo indicala bibliografía precedente (Cheldavi et al., 2014; Sam, Smith y Kai, 2017; PortelaPino, Bouzo-Gónzal ez \& Pino-Juste, 2019) el trabajo con caballos ha favorecido la mejora del equilibrio y por tanto la integración sensorial.

Finalmente podríamos concluir que se han cumplido el objetivo planteado previamente en la investiga ción y se considera que el cuestionario creado es una herramienta válida para la valoración del equilibrio sobre el caballo mediante la aplicación del programa descrito en lamismarúbrica, con alumnado conTEA, (Anexo 1).

Como hecho colateral, hemos podido comprobar en nuestro trabajo que coincidimos con Franquet (2017) cuando argumenta en su investigación que la intervención asistida con caball osse presenta como una propuesta psicoeducativa cuyos principales planteamientos peda gógicos están vinculados a la educación al aire libre.

A tenor de los resultados obtenidos convenimos con 
la autora en que este tipo de inter vención se constituye en un complemento a la educación formal, un complemento donde se ha favorecido la mejora de la integra ción sensorial y en consecuencia el equilibrio y el desa rrollo de habilidades a través de un aprendizaje vivencial aprovechando los recursos educativos que ofrece la na turaleza, entendiendo que el proceso educativo se puede llevar más allá de las cuatro paredes del aula.

Además de la mejora de las habilidades motrices y del equilibrio, montar a caballo puede mejorar al gunos de los síntomas centrales de losTEA como son los comportamientos ester eotipados. Existen algunas explica ciones que permiten apoyar esta afirmación. En primer lugar, el ejercicio físico proporciona un estado de relajación que mejora la ansiedad que suele estar en la base de las conductas estereotipadas (Sowa y M eulenbroek, 2012; García-Gómez et al., 2020); en segundo lugar, montar a caballo exige la puesta en marcha de conductas intencionales que requieren un alto grado de atención y el control ejecutivo de la conducta, lo cual supone un programa motor incompatible con las estereotipiasmotoras (Bass, Duchowny y Llabre, 2009; Yotchukiat, Pongsaksri y Peansu, 2016); en tercer lugar, la equita ción adaptada se realiza en un entorno natural multisensorial que facilita la integración sensorial, mejorando la tolerancia y las reacciones ante los inputs sensoriales (Ward et al., 2013), lo que facilitala adquisición de conductas adaptadas y la reducción de las estereotipias(Portela-Pino, I., Bouzo-Gónzalezy Pino-Juste, 2020; Gabriels et al., 2012); y, en cuatro lugar, montar a caba llo es una práctica individual y altamente motivadora por lo que se adapta de manera particular a las necesidades de las personas con autismo (García-Gómez et al., 2014).

Q uizá uno de los objetivos más ambiciosos ha sido el poder iniciar el protocolo para sentar las bases de lo que en un futuro podría ser un modelo de intervención educativa dirigido a la mejora del equilibrio y control postural en el que se proponga el trabajo de intervención asistida con caballos como complemento educativo realizado en un entorno natural. Sin embargo, a pesar de los interesantes resultados preliminares ofrecidos en el presente trabajo las conclusiones ofrecidas no pueden considerarse como definitivas ya que existen algunas limitaciones que es preciso señalar. Por un lado, la muestra es pequeña y tiene un sesgo de sexo, ya que está compuesta por ocho niños y una niña, y, por otro lado, las edades de los participantes oscilan entre los 9 y los 12 años, dejando de un lado ala participación en el programa a niños y niñas de otras edades. Los interrogantes que plantean estas limitaciones deberán ser resueltas en el futuro con trabajos que permitan aumentar la muestra de participantes, lo cual ofrecerá la posibilidad de realizar un análisis pormenorizado en función del género, de las edades, e incluso de los diversos niveles de afectación dentro del propio trastorno.

Además, ampliar la investigación teniendo en cuenta otras variables distintas al equilibrio, permitirá comprobar si los ejercidos propuestos en la rúbrica que hemos presentado, aporta otros benéficos en áreas propias del autismo como son los comportamientos estereotipados, el lenguaje y las habilidades de interacción.

\section{Referencias}

Adolph, K. E., \& Franchak, J. M. (2017). The development of motor behavior. Wiley Interdisciplinary Reviews: Cognitive Science, 8(1-2), e1430.

Akyol, B., \& Pektas, S. (2018). The Effects of Gymnastics Training Combined with Music in Children withAutism Spectrum Disorder and Down Syndrome. International Education Studies, 11(11), 45-51.

APA. American Psychiatric Association. (2013). Diagnostic and statistical manual of mental disorders, Fifth Edition, DSM 5. Arlington, VA, American PsychidricAssociation Ayres, A. J. (2008). La integración sensorial en los niños: Desafíos sensoriales ocultos ( 25 ed.). Madrid: TEA Ediciones, S.A.

Baio, J., Wiggins, L., Christensen, D. L., Maenner, M. J., Daniels, J., Warren, Z., ... \& Durkin, M. S. (2018). Prevalence of autism spectrum disorder among children aged 8 years - autism and developmental disabilities monitoring network, 11 sites, United States, 2014. MMWR Survéllance Summaries, 67(6), 1.

Bass, M. M., Duchowny, C.A., \& Llabre, M. M. (2009). The effect of therapeutic horseback riding on social functioning in children with autism. Journal of autism and developmental disorders, 39(9), 1261-1267.

Bronson, C., Brewerton, K., O ng, J., Palanca, C., \& Sullivan, S. J. (2010). Does hippotherapy improve balance in persons with multiple sclerosis: A systematic review. European Journal of Physical and Rehabilitation M edicine, 46, 347-353.

Charry-Sánchez, J. D., Pradilla, I., \& Talero-Gutiérrez, C. (2018). Effectiveness of animal-assisted therapy in the pediatric population: Systematic review and meta analysis of controlled studies. Journal of Developmental \& Behavioral Pediatrics 39(7), 580-590.

Cheldavi, H., Shakerian, S., Boshehri, S. N. S. \& Zarghami, M. (2014). The effects of balance training intervention 
on postural control of children with autism spectrum disorder: Role of sensory information. Research in Autism Spectrum Disorders, 8(1), 8-14.

Crespo-Eguílaz, N. y Narbona, J. (2009). Trastorno deaprendizaje procedimental: características neuropsicológicas. Revista de N eurología, 49(8), 409-416. https: / / doi.org/ 10.17979/ reipe. 2017.0.11.2218

Escobar-Pérez, J., \& Cuervo-martínez, A. (2008). Validez de contenido y juicio de expertos: una aproximación a su utilización. Avances en medición, 6, 27-36.

Fisher, A., Engel, C., Geist, R., Lillie, K., Lutman, S., \& Travers, B. G. (2018). Brief report: postural balance and daily living skillsin children and adolescents with autism. Journal of autism and developmental disorders, 48(9), 32103215.

Fleiss, J. L. (1971). Measuring nominal scale agreement among many raters. Psychological Bulletin, 76(5), 378382. https:/ / doi.org/ 10.1037/ h0031619

Fournier, K., Hass, C., Naik, S., Lodha, N. \& Cauraugh, J. (2010). Motor coordination in autism spectrum disorders: a synthesis and meta-analysis. Journal of autism and developmental disorders, 40(10), 1227-1240. https:/ / doi.org/ 10.1007/ s10803-010-0981-3

Francino Barrera, G. , JiménezTorres, S., Coloma Díaz, C., DelgadoVásquez, D., \&Verdugo Millar, D. (2019). Efectos de un programa de ejercicios de control postural en el equilibrio corporal y precisión de lanzamiento en tiro con arco en categoría infantil y cadetes. Retos, 37, 291296. https:/ / doi.org/ 10.47197/ retos. v37i37. 70956

Franquet, M a B. (2017). H ojarasca en otoño, amapolasen primavera, caballos todo el año. La intervención psicoeducativa asistida con caballos. un contexto educativo vivo más allà de las cuatro paredes. Juneda: Fonoll.

Gabriels, R. L., Pan, Z., Dechant, B., Agnew, J. A., Brim, N., \& Mesibov, G. (2015). Randomized controlled trial of therapeutic horseback riding in children and adolescents with autism spectrum disorder. Journal of theAmerican Academy of Child \& Adolescent Psychiatry, 54(7), 541-549.

Gabriels, R.L., Agnew, J.A., Holt, K.D., Shoffner, A., Zhaoxing, P., Ruzzano, S., Clayton, G.H. and M esibov, G. (2012). Pilot study measuring the effects of therapeutic horseback riding on school-age children and adolescents with autism spectrum disorders. Research In Autism Spectrum Disorders, 6, 578-588.

García-Gómez, A., Guerrero-Barona, E., GarcíaPeña, I., Rodríguez-J iménez, M., \& M oreno-M anso, J. M. (2020). Equine-assisted therapeutic activities and their influence on the heart rate variability: A systematic review. Complementary therapiesin clinical practice, 39, 101167.
GarcíaGómez, A., Rubio, J. C., Risco, M. L., Guerrero, E., \& GarcíaPeña, I. M. (2014). Efectos de un programa de equitación adaptada y terapéutica en un grupo de niños con trastornos del espectro autista. Electronic ournal of Research in Educational Psychology, 12(1), 107-128.

GaticaLara, F., \& U ribarren-Berrueta, T. D. N. J. (2013). ¿ Cómo elaborar una rúbrica?. Investigación en educación médica, 2(5), 61-65.

González, J., Sánchez, P., \& Jornet, J. m. (2011). Estudio de validación por jueces de una escala para medir la competencia de compromiso en universitarios. XV Congreso Nacional y I Internacional de M odelos de Investigación Educativa: Investigación y Educación en un mundo en red, Madrid.

H afner, J., \& H afner, P. (2003). Q uantitative analysis of the rubric asan assessment tool: an empirical study of student peer group rating. Int. J. Sci. Educ., 25(12), 15091528. Reddy,Y. M., \& Andrade, H. (2010). A review of rubric use in higher education. Assessment $\&$ evaluation in higher education, 35(4), 435-448.

Hameury, L., Delavous, P, Teste, B., Leroy, C., Gaboriau, J.C. and Bertithier, A. (2010). Équithérapie et autisme. Annales Médico-psychologiques, 168(9), 665-659. https:/ / doi.org/ 10.1016/ j.amp.2009.12.019

Jonsson,A. (2014). Rubricsasaway of providing transparency in assessment. Assessment \& Evaluation in Higher Education, 39(7), 840-852.

Kalsner, L., Twachtman Bassett, J., Tokarski, K., Stanley, C., Dumont Mathieu, T., Cotney, J., \& Chamberlain, $\mathrm{S}$. (2018). Genetic testing including targeted gene panel in a diverse clinical population of children with autism spectrum disorder: Findings and implications. M olecular genetics \& genomic medicine, 6(2), 171-185.

Kern, J. K., Fletcher, C. L., Garver, C. R., Mehta, J. A., Grannemann, B. D., Knox, K. R., ... \& Trivedi, M. H. (2011). Prospective trial of equine-assisted activities in autism spectrum disorder. AlternativeTherapies in $\mathrm{H}$ ealth \& M edicine, 17(3).

Lord, C., Rutter, M., Dilavore, P. C., Risi, S., Gotham, K., Bishop, S. L. ... y Guthrie, W. (2015). ADOS-2, Escala de observación para el diagnóstico del autismo. Madrid: TEA Ediciones.

M æenner, M. J., Shaw, K. A., \& Baio, J. (2020). Prevalence of autism spectrum disorder among children aged 8 years- autism and developmental disabilities monitoring network, 11 sites, U nited States, 2016. M MWR Surveillance Summaries, 69(4), 1.

Martínez-Rojas, J. G. (2008). Las rúbricas en la evaluación escolar: su construcción y su uso. Avances en medición, 6(129), 38.

Montes-Castillo, M. L., Benítez, M. P., Díaz-Barriga, A. S. y 
Jasso, A. V. (2000). El balance y las caídas en la tercera edad: consecuencias, evaluación y tratamiento. Revista Mexicana de medicina físca y rehabilitación, 12(1), 16-20. Disponible en https: / www.medigraphic.com/ pdfs/ fisical mf-2000/ mf001c. pdf (Consulta: 15 de noviembre de 2018)

Mosston, M. (1993). La enseñanza dela educación física. BarceIona: Paidós.

Munoz-Lasa, S., Ferriero, G., Valero, R., Gomez-M uniz, F., Rabini, A. \& \& Varela, E. (2011). Effect of therapeutic horseback riding on balance and gait of people with multiplesclerosis. Giornale Italiano Di M ediana Del Lavoro Ed Ergonomia, 33(4), 462-467.

Naksakul, P. (2008). Comparison of standing and walking balance between children with autism and typical children (Trabajo Final de Máster). Universidad de Mahidol, Tailandia. Disponible en: http:/ / dric.nrct.go.th/ direct fulltextphp?Bid=E40460yile=4737040_PTPT_M.pdf (Consultado: 21 de agosto de 2019).

Nosek, B. A., Aarts, A. A. , Anderson, J. E., Kappes, H. B., $\&$ O pen Science Collaboration. (2015). Estimating the reproducibility of psychological science. Science, 349 (6251), aac4716-aac4716.

Pan, C.Y., Chu, C. H., Tsai, C. L., Sung, M. C., Huang, C. Y., \& Ma, W.Y. (2017). The impacts of physical activity intervention on physical and cognitive outcomes in children with autism spectrum disorder. Autism, 21(2), 190-202.

Pollock, A. S., Durward, B. R., Rowe, P. J. y Paul, J. P. (2000). W hat is balance?. Clinical Rehabilitation, 14(4), 402-406. doi: 10.1191/ 0269215500cr3420a

PortelaPino, I., Bouzo-Gónzalez, S. \& Pino-Juste, M. (2019). Evaluation of an equine therapy program in students with Autism spectrum disorder. Journal of H uman Sport and Exercise, in press. doi:https:/ / doi.org/10.14198/ jhse. 2020.154.06

PortelaPino, I., Bouzo-Gónzalez, S., \& Pino-Juste, M. (2020). Evaluation of an equine therapy program in students with Autism spectrum disorder. Journal of Human Sport and Exercise, 15(4), 783-793.

Radonovich, K. J., Founier, K. A. y Hass, C. J. (2013). Relationship between postural control and restricted, repetitive behaviors in autism spectrum disorders. Frontiers in integrativeneuroscicence, 7 (28). doi: 10.3389/ fnint. 2013.00028

Randolph, (2008). O nline Kappa Calculator [Software informático]. http:/ / justus. randolph. name/ kappa

Randolph, J. J. (2005). Free-margina multirater kappa: An alternative to Fleiss' fixed marginal multirater kappa. Paper presented at the Joensuu University Learning and
Instruction Symposium 2005, Joensuu, Finland, 0 ctober 14-15th, 2005. (ERIC Document Reproduction Service No. ED 490661). Disponible en: https:// files eric. ed.gov/ fulltext/ ED 490661. pdf (Consultado: 9 de enero de 2020).

Reddy,Y. M., \&Andrade, H. (2010). A review of rubric use in higher education. Assessment \& evaluation in higher education, 35(4), 435-448.

Ricciardi, M. F. (2010). Sistema vestibular: el sentido del movimiento [M ensaje en un blog]. Disponible en: http:// integrandosensaciones. blogspot. mx/ 2010/ 04/ sistemavestibular-el-sentido-del.html (Consultado: el 18 de agosto de 2019)

Rutter, M., LeC outeur, A. y Lord, C. (2006). ADIR. Entrevista para el diagnóstico del autismo, edición revisada. Madrid:TEA Ediciones Lord, C., Rutter, M., DiLavore, P. C., Risi, S., Gotham, K., Bishop, S. L. ... y Guthrie, W. (2015). ADOS-2, Escala de observación para el diagnóstico del autismo. Madrid:TEA Ediciones.

Sam, A. M., Cox,A.W., Savage, M. N.,Waters, V., \& 0 dom, S. L. (2019). Disseminating information on evidencebased practices for children and Youth with Autism Spectrum Disorder: AFIRM. Journal of autism and developmental disorders, 1-10.

Sam, KL, Smith, AW y Kai, LS (2017). Cognición visual y equilibrio dinámico en personas con trastorno del espectro autista. Revista Internacional de Ciencias Sociales y Humanidades, 7 (5), 274-277.

Sarabzadeh, M., Azari, B. B. \& \& Helalizadeh, M. (2019). The effect of six weeks of Tai Chi Chuan training on the motor skills of children with Autism Spectrum Disorder. Journal of bodywork and movement therapies, 23(2), 284290.

Sowa, M., \& Meulenbroek, R. (2012). Effects of physical exercise on autism spectrum disorders: a meta-analysis. Research in autism spectrum disorders, 6(1), 46-57.

Stevenson, J., Lindley, C. y M urlo, N. (2017). Retrospectively assessed early motor and current pragmatic language skills in autistic and neurotypical children [Evaluando retrospectivamente las habilidades motoras tempranas y actuales en niños autistas y neurotípicos]. Perceptual and motor skills, 124(4), 777-794. https:/ / doi.org/ 10.1177/ 0031512517710379

Tabares, C., Vicente-Castro, F. V., \& Sánchez-Herrera, S. (2013). Análisis del cambio hormonal en una población de niños autistas por el efecto de la terapia asistida con caballos. International Journal of Developmental and Educational Psychology, 1(1), 615-621.

Travers, B. G., Mason, A. H., Mrotek, L. A., Ellertson, A., Dean, D. C., Engel, C., ... \& McLaughlin, K. (2018). 
Biofeedback-based, videogamebalancetraining in autism. Journal of autism and developmental disorders, 48(1), 163175.

Travers, B. G., Powell, P. S., Klinger, L. G. y Klinger, M. R. (2013). Motor difficulties in autism spectrum disorder: linking symptom severity and postural stability. Journal of autism and developmental disorders, 43(7), 1568-1583. doi: 10.1007/ s10803-012-1702- $x$

Trzmiel, T., Purandare, B., Michalk, M., Zasadzka, E., \& Pawlaczyk, M. (2019). Equine assisted activities and therapies in children with autism spectrum disorder: A systematic review and a metaranalysis. Complementary therapies in medicine, 42, 104-113.

Tseng, S. H., Chen, H. C., \&Tam, K.W. (2013). Systematic review and metaznalysis of the effect of equine assisted activities and therapies on gross motor outcome in children with cerebra palsy. Disability and Rehabilitation, 35(2), 89-99.

Villalobos-Samaniego, C., RiveraSosa, J., Ramos-jimenez, A., Cervantes-Borunda, M., Lopez-Alonzo, S., \& Hernandez-Torres, R. (2019). Métodos de evaluación del equilibrio estático y dinámico en niños de 8 a 12 años (Evaluation methods of static and dynamic balance in children aged 8 to 12 years old). Retos, 37, 793-801. https:/ / doi.org/ 10.47197/ retos. v37i37.67809

Ward, S. C., W halon, K., Rusnak, K., Wendell, K., \& Paschal, N. (2013). The association between therapeutic horseback riding and the social communication and sensory reactions of children with autism. Journal of autism and developmental disorders, 43(9), 2190-2198.

Warrens, M. J. (2010). Inequalitiesbetween kappaand kappa like statistics for kx k tables. Psychomerrika, 75(1), 176185. doi.org/ 10.1007/ s11336-009-9138-8

W HO (2019). The ICD-11 Classification of Mental and Behavioural Disorders. Clinical descriptions and diagnostic guidelines. Disponible en: https:/ / www.who.int/ classifications/ icd/ en/ (Consultado: 29 dejunio de2020) W illiamson, E., Sathe, N.A.,Andrews, J. C., Krishnaswami, S., M cPheeters, M. L., Fonnesbeck, C., ... \& Warren, Z. (2017). M edical therapies for children with autism spectrum disorder-An update. Comparative Effectiveness Review No. 189. Agency for Healthcare Research and Quality. https:// doi.org/ 10.23970/ AHRQEPCCER189.

Yotchukiat, S., Pongskksri, M., y Peansu, S. (2016). Effects of Equine Assisted Therapy on Response Reaction Time and Attention Span of Youthswith Autistic Spectrum Disorder. International Journal of Child Devlelopment and Mental $H$ ealth, 4(1), 38-48.
ANEXO 1

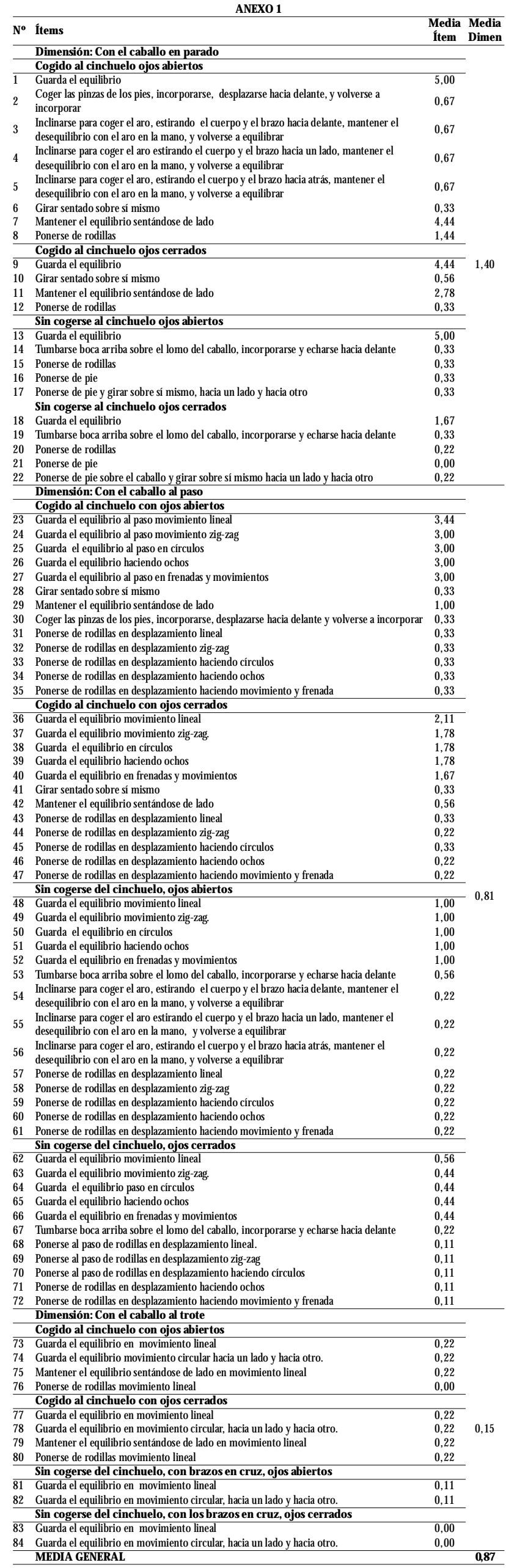

